\title{
Flow-Induced Twist-Compression in a Twisted Nematic Cell
}

\author{
L. Z. Ruan and J. R. Sambles \\ Thin Film Photonics, School of Physics, University of Exeter, Stocker Road, Exeter EX4 4QL, United Kingdom
}

(Received 19 September 2002; published 25 April 2003)

\begin{abstract}
An optical convergent-beam guided-wave technique is used to explore in detail the dynamic flow effects in a twisted nematic cell. During switch-on it is found that the dynamic flow compresses the director twist to regions close to the cell walls. For high fields this twist compression takes the cell far beyond the Mauguin limit and it no longer effectively guides the polarization of the light through the cell. This results in a very fast switch to a transient dark state.
\end{abstract}

DOI: 10.1103/PhysRevLett.90.168701

Nematic liquid crystals form the basis of the majority of flat screen displays. Their switching dynamics, key to their functionality as video displays, is understood in terms of the foundational theory pioneered by Ericksen [1,2] and Leslie [3]. Developed in the 1960s, and simplified by Berreman and van Doorn [4,5], this theory of flow in anisotropic fluids is still very widely used. However, there exist very few detailed experimental confirmations of many of its predictions. This is particularly true as regards to switch-on dynamics where the influence of flow in a relatively fast, field-driven process has barely been explored. Some simple experiments [6,7] have confirmed the theoretically predicted "backflow" in a twisted nematic (TN) cell during switch-off by recording an optical "bounce" effect in transmission. However, almost all the experiments [8] are based on simple transmission or reflection observations during switching and as such the signals from the liquid crystal cell are an integrated response. Only recently, using a convergentbeam optical guided-wave technique $[9,10]$, have details of the director profile in the liquid crystal cell during switch-off been unambiguously obtained, thereby establishing the validity of the theory. Further there has been no published work which details the change of director profile within a cell during switch-on, a much faster process providing a more rigorous test of the theory.

In this present study a convergent-beam system is used to investigate the switch-on dynamics of a twisted nematic cell detailing directly the strong influence of flow on the twist profile, and, in particular, the strong compression of director twist to the cell walls.

The liquid crystal cell comprises two low index glass plates $(n=1.5170$ at $632.8 \mathrm{~nm})$, each coated inside by a thin ( $\sim 50 \mathrm{~nm}$ ) layer of indium tin oxide (ITO), on top of which are surface aligning layers of rubbed polyimide. The plates are assembled together with $6.0 \mu \mathrm{m}$ spacers in between. A twisted director profile is established by setting the angle between the rubbing directions of the upper and lower plates to about $87^{\circ}$. The cell is filled with liquid crystal ZLI-2293 (Merck) in the isotropic phase and then slowly cooled down to room temperature forming a monodomain.
PACS numbers: 83.80.Xz, 47.17.+e, 42.79.Gn, 78.20.-e

The cell is then inserted between the two glass hemispheres, optical contact being achieved with matching fluid. The glass hemispheres and the matching fluid have the same index as the glass substrates of the liquid crystal cell. This complete assembly is then placed so that the center of the sample is at the focus of the laser beam. A schematic is given in Fig. 1. The He-Ne laser beam, of wavelength $632.8 \mathrm{~nm}$, is collimated into a less-coherent parallel beam of diameter about $5 \mathrm{~cm}$ by using one rotating and one fixed diffuser and a beam expander [9]. This collimated beam passes through a polarizer, a horizontal slit aperture, and a pair of converging lenses, being focused to a spot at the center of the cell to be studied. Both reflectivity and transmissivity signals from the cell are recorded through a polarizer by a charge-coupled device (CCD) camera (DALSA).

To obtain data most sensitive to the director profile in the cell it is set such that the rubbing direction at the surface has an angle of about $45^{\circ}$ with the incident plane. In addition, theoretical modeling indicates that the angular region of the fully leaky guided modes which is most sensitive to the director alignment is in the high in-plane wave vector area. To explore this angular region the cell is set such that the angle between the central axis of the convergent beam and the cell normal is $70^{\circ}$. This then gives optical information over an internal (in the hemispheres) angle range from $\sim 62^{\circ}$ up to $\sim 78^{\circ}$. Data may be taken over this range with a temporal resolution of $0.2 \mathrm{~ms}$.

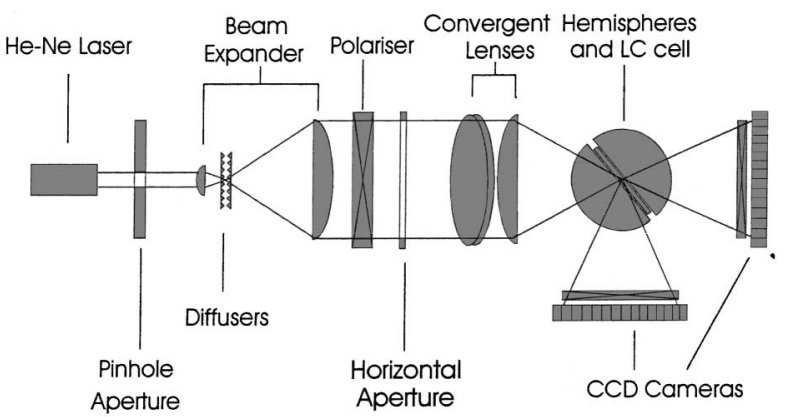

FIG. 1. The experimental setup for the convergent beam system. 
Choice of input polarizer ( $p$, transverse magnetic, or $s$, transverse electric) and output polarizer allows a variety of angle dependent signals to be recorded, including the polarization conserving reflectivities $R_{p p}$ and $R_{s s}$, polarization converting reflectivities $R_{p s}$ and $R_{s p}$, polarization conserving transmissivities $T_{p p}$ and $T_{s s}$, as well as polarization conversion transmissivity signals $T_{p s}$ and $T_{s p}$. Data are recorded with no voltage applied and then switch-on dynamic data are recorded under application of different ac $(10 \mathrm{kHz})$ fields applied across the cell. Figure 2 shows how one of the recorded signals, $T_{p p}$, varies with time at three different applied voltages. These figures show a dramatic change in the guided mode structure during switch-on. Complete switch-on times at voltages (rms) of 2.9, 4.7, and $6.8 \mathrm{~V}$ are $\sim 72$, $\sim 26$, and $\sim 11 \mathrm{~ms}$, respectively.

Using multilayer optical theory [11] to fit the reflection and transmission data the full director profile in the cell is obtained. From the data taken with no applied field the optical parameters of the different layers in the cell at $632.8 \mathrm{~nm}$ are first found as follows. The ITO layers have a thickness of $49 \mathrm{~nm}$ with an optical permittivity of $\varepsilon=$ $3.1790+i 0.15$, while the polyimide layers have anisotropic optical permittivities $\varepsilon_{\perp}=1.8015+i 0.001, \varepsilon_{\|}=$ $2.3010+i 0.001$ with a thickness of $29.5 \mathrm{~nm}$. The optical permittivities of the liquid crystal, ZLI-2293, are $\varepsilon_{\perp}=$ $2.2409+i 0.0003, \varepsilon_{\|}=2.6643+i 0.003$ with the thickness of the liquid crystal layer being $6.00 \mu \mathrm{m}$. The director uniformly twists through the whole cell from the top to the bottom through some $85.56^{\circ}$ with surface tilts of about $2.5^{\circ}$ on one surface and $0.5^{\circ}$ on the other. (This difference in surface pretilts arises from minor differences in the rubbing treatment of the two surfaces.)
After long-time application of an ac voltage the director structure in the cell is substantially altered. Based on the Frank-Oseen elasticity theory model director profiles to fit the data taken at $2.9,4.7$, and $6.8 \mathrm{~V}$ are also found. At these voltages, for most of the cell, the director is almost homeotropically aligned. This decreases the influence of the eleastic twist, allowing the director to return to nearer the original "easy" alignment axes on the surfaces. The director twist between the two surfaces is found to consequently increase to $87.56^{\circ}$, $88.30^{\circ}$, and $88.66^{\circ}$ for applied voltages of $2.9,4.7$, and $6.8 \mathrm{~V}$, respectively.

The dynamic data were analyzed by using a modeling program (DIMOS) based on the Ericksen-Leslie hydrodynamic theory [1-3] using the approximation of Berreman and van Doorn [4,5]. This means that in this modeling the fluid inertia is ignored and flow is restricted to the plane of the cell. The elastic constants, the dielectric and optical permittivities, the director surface angles, and the cell thickness are set from the preceding fits to static data.

The dynamical data were fitted for all times with single values of the viscosity coefficients $\gamma, \eta_{1}$ and small variations in the twist and tilt angles at the cell walls. This gave in detail the director profiles at different times. From the fitting of data taken over a long time (about $60 \mathrm{~ms}$ ) the viscosities $\gamma$ and $\eta_{1}$ are determined as 0.131 and $0.162 \mathrm{Pas}$, which are the same as those determined from switch-off dynamics of a twist cell [12]. They are a little smaller than the values given by Armitage and Larimer [13]. We also noted that there is a small change of director tilt at the cell surfaces during the dynamic switching.
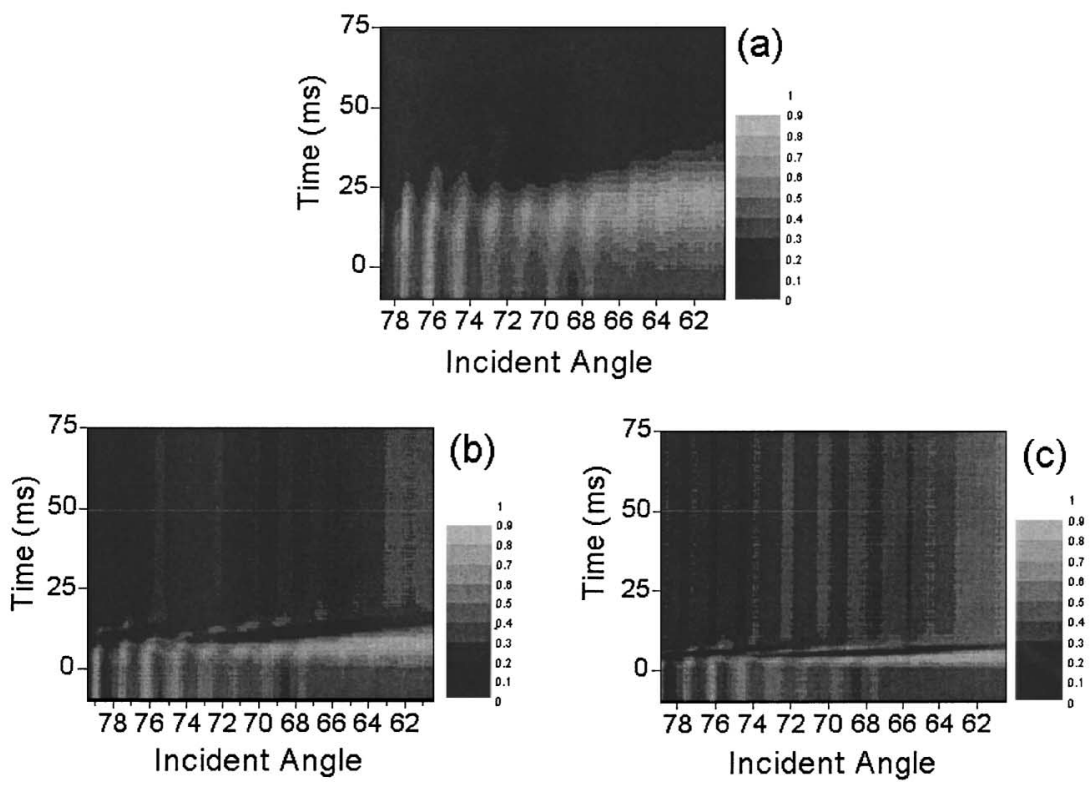

FIG. 2. The dynamic data of $T_{p p}$ after switch-on of an applied electric field to (a) $2.9 \mathrm{~V}$, (b) $4.7 \mathrm{~V}$, and (c) $6.8 \mathrm{~V}$. 

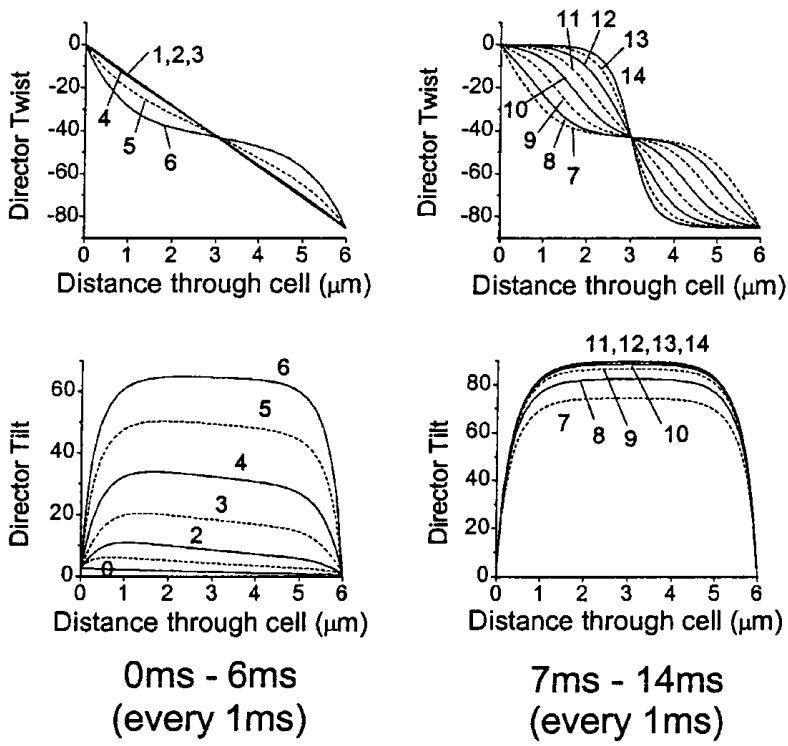

FIG. 3. Fitted director profiles at different times after switchon of $6.8-\mathrm{V}$ ac to the cell.

The most striking behavior of the cell dynamics is illustrated by the data taken at $6.8 \mathrm{~V}$ as shown in Fig. 3 . During the initial $4 \mathrm{~ms}$ the director twists linearly in the cell. After $5 \mathrm{~ms}$ this twist profile starts to change to a dramatically different form to that found in the cell either at zero volts or under a steady ac field. During these intermediate times $(5-8 \mathrm{~ms})$ the director twists are concentrated near to the surfaces of the cell. From 6 to $8 \mathrm{~ms}$ a region forms in the center of the cell having almost uniform director twist with only the director tilt varying. This region occupies nearly half of the cell. After $8 \mathrm{~ms}$ the director twist in the cell has a tendency to return to linearity. Then after $\sim 10 \mathrm{~ms}$ the twist profile swiftly changes to become much as that found in the cell with the steady applied field; that is, the change of the director twist is concentrated in the center of the cell.

Thus it is clear that in the switch-on dynamics there is an unusual behavior in the director twist profiles over a certain time period, where the changes of the director twists concentrate near the cell surfaces. The higher the applied voltage is, the more obvious this unusual phenomenon.

By analyzing the flow velocity in the dynamic process the above phenomenon may be explained. Figure 4(a) shows the model profiles (computed with the parameters obtained from the above fits to the guided mode data) of the components of the flow velocity in the cell plane, $V_{x}$ and $V_{y}$ through the cell, at different times after switching on $6.8 \mathrm{~V}$. After applying the electrical field to the TN cell, at the early stages $(0-3 \mathrm{~ms})$ the flow velocity is small, influencing little the director twist, which remains largly linear through the cell. At the same time the director tilt increases due to the effect of the electrical field. Sometime later (at about 4-6 ms) the flow velocity in the cell plane has increased [see Fig. 4(a) for $5 \mathrm{~ms}$ ] and starts to influence the director twist. Consideration of the director twist and velocity profiles at this time [see Fig. 4(b)] reveals that near to both cell surfaces there is an angle between the director and the flow direction. Because of this angle the fluid motion will cause the director to increase the gradient of twist away from the easy axis on the surfaces. This is why the director twist is concentrated near to the cell walls over this time scale. By contrast, in the middle of the cell the fluid flow has the same direction as the director orientation [very clearly visible in Fig. 4(b)], keeping the director unchanged. After about $12 \mathrm{~ms}$ the flow velocity has become quite small, ceasing to significantly influence the director. However, by this time the director in the middle of the cell has a large tilt angle and so the elastic coupling between the two surface anchoring forces has been broken. Therefore the directors close to the cell surfaces now tend to reorient parallel to the easy axes of the cell surfaces and the twist moves back to being in the center of the cell, away from the walls.

In conclusion, using the convergent-beam system together with the fully leaky guided-mode technique it has

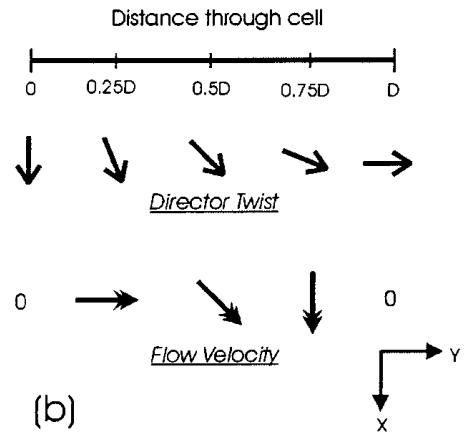

FIG. 4. Models using the fitted parameters for the cell of (a) the velocity profile at different times after switch-on of 6.8-V ac to the cell. (b) The relationship between the director twist and the velocity profile at $5 \mathrm{~ms}$. 
been possible to examine in detail for the first time the director switch-on process of a twisted nematic cell. The approximation of Berreman and van Doorn of the Ericksen-Leslie theory of the flow in the cell accords well with the data. There are only two viscosity coefficients, $\gamma$ and $\eta_{1}$, which play important roles in the switch-on dynamics, taking for the material ZLI2293 values of $\gamma=0.131 \mathrm{Pas}$ and $\eta=0.162 \mathrm{Pas}$. The switch-on processes for different applied voltages have been analyzed and the influence of flow on the director twist profile is particularly significant. For the highest applied fields the director flow associated with the increased tilt towards the cell center couples to the director in such a manner as to increase the twist rate near to the cell walls, effectively compressing the twist to within a region of about $1 \mu \mathrm{m}$. This director twist is now so rapid that polarization guiding (which is good but imperfect initially as the cell is actually already below the Mauguin limit) is almost completely destroyed, and between crossed polarizers the cell appears dark. This clearly shows how the combination of low frequency dielectric anisotropy (responding to the applied external field), the elastic constants of the liquid crystal, and its flow properties act together to control the dynamic director profile and optical response of such cells. It also convincingly confirms the validity of the Ericksen-Leslie theory for driven switch-on liquid crystal dynamics.
The authors acknowledge the Engineering and Physical Sciences Research Council of the U.K. for funding this research.

[1] J. L. Ericksen, Arch. Ration. Mech. Anal. 4, 231 (1960).

[2] J. L. Ericksen, Trans. Soc. Rheol. 5, 23 (1961).

[3] F. M. Leslie, Q. J. Mech. Appl. Math. 19, 357 (1966); Arch. Ration. Mech. Anal. 28, 265 (1968); Advances in Liquid Crystals, edited by G. H. Brown (Academic Press, New York, 1979), Vol. 4, p. 1.

[4] D.W. Berreman, J. Appl. Phys. 46, 3746 (1975).

[5] C. Z. van Doorn, J. Appl. Phys. 46, 3738 (1975).

[6] C. J. Gerritsma, C. Z. van Doorn, and P. van Zanten, Phys. Lett. 48A, 263 (1974).

[7] F. Nakano, H. Kawakami, H. Morishita, and M. Sato, Jpn. J. Appl. Phys. 19, 659 (1980).

[8] O. Cossalter and D. A. Mlynski, Liq. Cryst. 19, 545 (1995).

[9] N. J. Smith and J. R. Sambles, J. Appl. Phys. 85, 3984 (1999).

[10] N. J. Smith and J. R. Sambles, Appl. Phys. Lett. 77, 2632 (2000).

[11] D. Y. K. Ko and J. R. Sambles, J. Opt. Soc. Am. A 5, 1863 (1988).

[12] L. Z. Ruan and J. R. Sambles, J. Appl. Phys. 92, 4857 (2002).

[13] D. Armitage and J. Larimer, SID 96 Digest 584, 45 (1996). 